\title{
Routine immunohistochemical staining for high-molecular weight cytokeratin $34-\beta$ and $\alpha$-methylacyl CoA racemase (P504S) in postirradiation prostate biopsies
}

\author{
Mildred B Martens and Jeremy H Keller \\ Departments of Pathology, University of Western Ontario and London Health Sciences Centre, London, \\ ON, Canada
}

\begin{abstract}
A total of 43 cases of postirradiation prostate cores were assessed in an attempt to determine if routine use of $\alpha$-methylacyl-CoA racemase (AMACR) in conjunction with high-molecular weight cytokeratin (HMWCK) would increase the recognition of carcinoma in postirradiation prostate biopsies. We concluded that in most cases the addition of AMACR in conjunction with HMWCK does not increase the recognition of prostatic adenocarcinoma, however it is supportive in nature. In one case the use of AMACR highlighted the extent of the adenocarcinoma which otherwise would have been designated as atypical small acinar proliferation (ASAP). Further evaluation is required to assess the significance of a diagnosis of atypical small acinar proliferation in postirradiation prostate biopsies.
\end{abstract}

Modern Pathology (2006) 19, 287-290. doi:10.1038/modpathol.3800531; published online 9 December 2005

Keywords: $\alpha$-methylacyl-CoA racemase; AMACR; immunohistochemistry; P405S; postradiation; prostate biopsy

Postradiotherapy prostate needle cores present a diagnostic challenge in the identification of residual or recurrent prostate adenocarcinoma because there is often cytological atypia secondary to therapy. A diagnosis of malignancy is usually based on architectural features of prostatic adenocarcinoma in these patients. Recent availability of $\alpha$-methylacylCoA racemase (AMACR) as a positive marker of prostatic adenocarcinoma may make the diagnosis of recurrent cancer easier. In our study, 43 cases of postirradiation prostate cores that were initially signed out as negative for malignancy were processed with high-molecular weight cytokeratin $34-\beta$ (HMWCK) and AMACR; in an attempt to determine if the routine use of AMACR in conjunction with HMWCK increases the recognition of adenocarcinoma in postirradiation prostate cores.

A literature search using the terms 'P504S, racemase, radiotherapy' resulted in 1 paper by Yang et al, published in $2003^{1}$ that used AMACR in the evaluation of postirradiation cores. In this paper, 40 specimens were obtained; 25 after salvage radical

Correspondence: Dr M Martens, BSc, MD, FRCPC, Department of Pathology, University of Western Ontario and London Health Sciences Centre (LHSC), University Campus, 339 Windermere Road, London, ON, Canada N6A 5A5.

E-mail: mildred.martens@lhsc.on.ca

Received 13 September 2005; revised 20 October 2005; accepted 24 October 2005; published online 9 December 2005 prostatectomy, four after transurethral resection and 11 needle biopsies. In all, 28 of the 40 postirradiation specimens contained adenocarcinoma. All 28 specimens showed strongly positive AMACR immuno-staining in the areas of adenocarcinoma, identical to nonirradiated specimens. Irradiated benign glands adjacent to adenocarcinoma showed no immunoreactivity to AMACR even though cytologic atypia was present. These findings were supported by Beach et $a l^{2}$ who found four of five irradiation treated carcinomas showed immunopositivity with AMACR. Our study differs in that we are not attempting to determine if AMACR shows immunopositivity in postirradiation prostatic adenocarcinoma but rather if the routine use of AMACR in postirradiation cores is justified.

\section{Materials and methods}

Our protocol was reviewed and approved by the local Institutional Review Board. Patient consent was provided for review of slides and access to clinical information. The database of surgical reports, between the years 1995 and 2000 at the London Health Sciences Centre, was searched using the terms 'prostate biopsy/biopsies' and 'radiation'. Cases were excluded if they were diagnosed as positive for malignancy or if there had been prior treatment with cryotherapy. Three levels had been 
originally cut from the paraffin blocks. The original slides were reviewed and specimens were excluded if tissue was composed of bowel wall without prostatic glandular tissue or if there was insufficient residual tissue in the corresponding paraffin blocks. In total, 43 patients were included in this study. Four cases included cores, which had been originally designated as ASAP and were included in our study as these foci were not convincing for invasive adenocarcinoma. These 43 patients were designated sequently according to the date of their reports. Two to eight suitable blocks were available from any given case, for a total of 235 blocks.

Four slides were cut from each formalin-fixed, paraffin-embedded archival block. Given the limited amount of residual tissue in these archival blocks only a single section was placed on each slide. The first and second sections were stained for highmolecular weight cytokeratin 34bE12 (HMWCK) (DakoCytomation) and AMACR/p504S (Zeta Corporation). Antigen retrieval was performed with prewarmed $10 \mathrm{mM}$ citrate buffer in a $1250 \mathrm{~W}$ microwave for $3 \mathrm{~min}$ on high power then $22 \mathrm{~min}$ on low power followed by 5 min no power. Immunohistochemistry was carried out on a Dako Autostainer. Sections were biotin blocked with Avidin D solution (Vector). Sections were then incubated with either mouse monoclonal HMWCK or rabbit monoclonal AMACR/p504S with a 1:50 dilution, with a biotin solution (Vector) for $1 \mathrm{~h}$. Sections were incubated in biotinylated secondary antibody (Vector) and Vectastain Elite ABC kit (Vector) for $30 \mathrm{~min}$ each, followed by development in DAB (BioGenex). Sections were counterstained with Gill's Hematoxylin. Using the standard technique, H\&E slides were prepared on the 3rd section cut from each block. The 4th section was used as a negative immunohistochemical control.

\section{Results}

All newly cut H\&E-stained slides were assessed prior to evaluation of the immunohistochemistry slides. These were assigned a grade of 'negative for adenocarcinoma, atypical small acinar proliferation (ASAP), or diagnostic for malignancy'. The corresponding HMWCK and AMACR slides were then examined to see if the findings supported our H\&Ebased diagnosis, as well as to detect any foci of adenocarcinoma that might have been missed on examination of the H\&E-stained slides alone. Specimens were designated as 'diagnostic for malignancy' if there were areas of architecturally abnormal glands, the HMWCK was negative and the AMACR was positive. The intensity of the AMACR immunopositivity was variable but considered to be convincing when there was circumferential, granular, apical or diffuse cytoplasmic staining. Architecturally atypical foci composed of less than four to five glands, lacking a basal cell layer on examination of the HMWCK and which showed convincing immunopositivity with AMACR, were designated as ASAP. Adenocarcinoma was identified in patients \#15, \#32 and \#40.

In patient \#15, prostatic adenocarcinoma was identified in 1 core. The original slides showed a focus of ASAP and had been signed out as 'focal glandular atypia consistent with radiation changes'. The adenocarcinoma was identified on the newly recut H\&E-stained slide, being composed of about 50 small acini. Recognizing that grading prostatic adenocarcinoma after radiotherapy treatment may not be reliable, this focus had an apparent Gleason score of $3+3=6 / 10$. HMWCK was confirmatory in that there was no evidence of a basal cell layer. AMACR showed circumferential luminal cytoplasmic staining in all neoplastic glands.

In patient \#32, prostatic adenocarcinoma was identified in two of the cores, part C and E. Part C of this case had been originally signed out as 'atrophic changes with focal atypia, negative for malignancy'. On review of the original slides, two atypical acini were identified but it was only on examination of the AMACR that the extent of the prostatic adenocarcinoma was realized. There was strong, diffuse granular cytoplasmic positivity which highlighted numerous single cells, having an apparent Gleason score of $5+3=8 / 10$ (see Figure 1). Core $\mathrm{E}$ of this sextant biopsy case contained a focus of glands in 1 core, which had originally been signed out as 'ASAP'. Deeper sections from this block showed approximately 50 small irregular glands, which were negative for AMACR, having an apparent Gleason score of $3+3=6 / 10$. A basal layer was not identified in either of these cores. The AMACR was repeated with the same negative result.

In patient \#40, prostatic adenocarcinoma was identified in 1 core. The original slides showed no evidence of malignancy. Prostatic adenocarcinoma was identified on the newly recut H\&E-stained slides, being composed of about 20 fused glands with several adjacent small atypical acini, having an apparent Gleason score of $4+3=7 / 10$. HMWCK were confirmatory in that there was no evidence of a basal cell layer. AMACR showed circumferential luminal cytoplasmic staining in the neoplastic glands.

ASAP was identified in 16 additional patients. These foci consisted of small groups of two to four acini, showing no evidence of a basal layer with HMWCK and showing convincing immunopositivity with AMACR. Glands having the architectural features of LGPIN or HGPIN frequently showed strong apical to diffuse cytoplasmic staining.

All cases had clinical follow-up of no $<4$ years. Hospital records were searched for clinical evidence of recurrences of prostatic adenocarcinoma. Any pre- and postbiopsy clinical testing, cryotherapy or radiotherapy interventions and any surgical interventions including outcome was recorded. The cause of death if applicable was recorded. Two of 


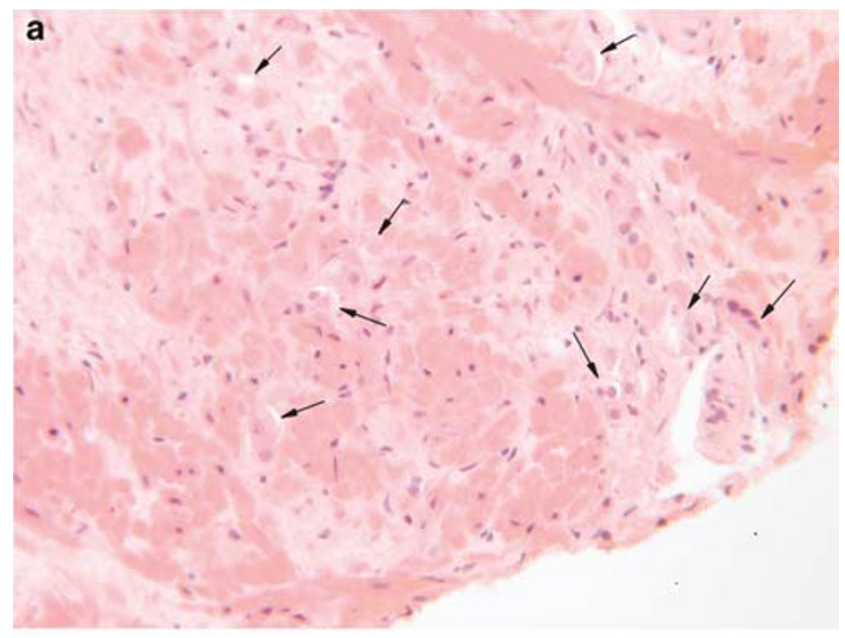

b

Figure 1 (a) Core 32C H\&E $\times 250$; (b) Core $32 \mathrm{C}$ AMACR $\times 250$.

the three patients (\#15, \#32) who had a missed diagnosis of adenocarcinoma in the study cores had subsequent positive cores. The third patient (\#40) was subsequently diagnosed with metastatic bone disease.

\section{Discussion}

Postradiotherapy prostate needle cores present a diagnostic challenge in the identification of residual or recurrent prostatic adenocarcinoma because there is often cytological atypia in both the benign and malignant glands, secondary to therapy. Often there is more atypia in the benign glands than in the irradiated malignant glands. Malignant glands may take two forms postradiation therapy.

In some the cancer appears unaffected by radiation and resembles nontreated adenocarcinoma of the prostate. In other cases, which are more difficult to diagnose, the cancer shows treatment affect. This appears to be the case in the core we have illustrated in Figure 1. Diagnosis can be further complicated if there is associated inflammation and architectural distortion of glands. Diagnosis of malignancy in postradiotherapy cores is usually based on architectural features of prostatic adenocarcinoma. A 2003 review paper by Evans et $a l^{3}$ states 'studies to date with AMACR/P504S clearly demonstrate the ability of this marker to support a diagnosis of malignancy in prostate needle cores. This is particularly true, when it is combined with negative staining for a basal cell marker.' He prefaces this statement with a comment that 'the diagnosis of prostatic adenocarcinoma remains dependent on the recognition of hematoxylin and eosin criteria'. The acceptable H\&E criteria is defined as acini that infiltrate prostatic stroma, show nuclear atypia with or without macronucleoli and lack a basal cell layer.

HMWCK when demonstrating a focal or diffuse basal layer, is helpful in designating glands which are 'concerning for malignancy' as benign in nature. However, HMWCK is a negative stain and there are well-recognized inherent problems with a negative stain. Assessment for the presence of a basal layer has several pitfalls. Benign mimics of prostatic adenocarcinoma (HGPIN, partial atrophy, postatrophic hyperplasia, and atypical adenomatous hyperplasia (AAH)/adenosis) often have a discontinuous basal cell layer. Prolonged formalin fixation has been shown to have a negative effect on detection of basal cell-specific keratins giving rise to false negative staining. ${ }^{3}$ Some morphological variant of prostatic adenocarcinoma, most notable being prostate duct adenocarcinoma, may show focally positive basal cell staining. An internal control should be present in the form of adjacent benign glands demonstrating a basal layer with HMWCK. Finally and significantly, HMWCK does not aid in the detection of small foci of adenocarcinoma, as inherently adenocarcinoma lacks a basal layer, whereas the use of AMACR/P504S might.

Recent studies have shown that AMACR expression is positive in a variety of prostatic lesions including high-grade prostatic intraepithelial neoplasia, AAH, benign prostatic hyperplasia and adenocarcinoma. $^{3}$ Several published studies $^{2,4,5}$ have specifically dealt with this sensitivity and specificity question in needle cores, showing sensitivities that range from 82 to $100 \%$ and specificities ranging from 79 to $100 \%$. The intensity of the stain ranged from weak to strong independent of the Gleason score and fixative technique. Beach et $a l^{2}$ evaluated the distribution pattern and found that circumferential luminal to subluminal or diffuse staining were the most specific patterns for prostatic adenocarcinoma. It should be remembered that $2-$ $21 \%$ of benign glands stain with AMACR. ${ }^{2,4}$ Staining intensity in the benign glands ranged from weak to strong but in most cases was weak, luminal and noncircumferential. To be convinced of a true positive stain, the intensity should be stronger and more diffuse than that which is seen in the adjacent obviously benign glands. 
In our study, we diagnosed invasive prostatic adenocarcinoma in 4 cores from three patients, all of which had been originally reported as negative for malignancy. In three of these cores a positive diagnosis was made after examination of the recut H\&E-stained slides. In the fourth core two small acini were identified on H\&E-stained slides, however, it was only when the AMACR was reviewed that the extent of the adenocarcinoma was realized. The areas of carcinoma were negative for HMWCK in all 4 cores. AMACR staining was circumferential and luminal in patient \#15 and \#40. In patient \#32 prostatic adenocarcinoma was identified in 2 of the cores. The first core had an apparent Gleason score of $3+3=6 / 10$ and interestingly was totally negative for AMACR, while the second core had an apparent Gleason score of $5+3=8 / 10$ and showed strong diffuse granular cytoplasmic positivity (see Figure 1). The AMACR was repeated a second time with the same results. Beach et $a l^{2}$ found that $18 \%$ of adenocarcinomas lacked immunopositivity. Evans ${ }^{3}$ suggested that a major factor contributing to this variability could be the different anti-AMACR/ P504S antibodies used in these studies, different concentrations of the primary antibodies, manual vs automated staining techniques, and different protocols for antigen retrieval. These hypotheses do not explain why in case \#32, a focus of adenocarcinoma in one core was negative for AMACR, while a second focus in a separate core from the same patient showed strong diffuse cytoplasmic positivity. Beach et $a l^{2}$ stated that the Gleason score did not correlate with staining intensity, but did not comment specifically on the distribution of grades in the adenocarcinomas which were negative for AMACR.

ASAP was identified in 16 patients or $37 \%$ of all patients in our study. ASAP was diagnosed if architecturally atypical foci were composed of $<4-5$ glands, lacked a basal cell layer on HMWCK and showed diffuse or apical staining with AMACR. We hesitated to make a definite diagnosis of adenocarcinoma when $<5$ atypical glands were present even when there was convincing AMACR staining. We could not be sure this did not represent a missampling of a patchy basal cell layer that is often seen in benign mimics of prostatic adenocarcinoma which can be positive for AMACR, such as AAH and HGPIN. This is supported by several studies, ${ }^{2,4}$ which showed that $20-80 \%$ of cases of HGPIN showed granular intracytoplasmic pattern with AMACR. Yang et $a l^{5}$ showed that in seven of 40 $(18 \%)$ examples of AAH there was detectable staining for AMACR, being focal in four of 40 and diffusely positive in three of 40 , concluding that AMACR immunostaining distinguishes most but not all cases of AAH from adenocarcinoma.

In summary, three of the 43 patients in our study had a revised diagnosis of adenocarcinoma based on
H\&E appearance. This was supported by the lack of a basal layer as assessed with HMWCK. One of the three patients had two cores positive for adenocarcinoma. In three of the four positive cores, a correct diagnosis would have been made if deeper levels had been examined. In the final positive core the diagnosis was made only with a confirmatory AMACR stain. In all, 16 of 43 patients had a revised diagnosis of ASAP. These glands were small, the HMWCK was negative and the AMACR showed convincing positivity. The numbers of glands were too limited to be confident they did not represent mis-sampling of a patchy basal cell layer in areas of AAH or HGPIN.

Based on this study, we concluded that in most cases the addition of AMACR in conjunction with HMWCK does not increase the recognition of prostatic adenocarcinoma, however, it is supportive in nature. Specifically, the addition of AMACR in conjunction with HMWCK does not identify small foci of cancer missed on H\&E-stained sections. In 1 core the use of AMACR highlighted the extent of the adenocarcinoma which otherwise would have been designated as ASAP. More research is required to determine the minimum number of glands required for a definite diagnosis of adenocarcinoma, even when the glands show convincing immunopositivity for AMACR. The finding of lack of positivity for AMACR in 1 core while a second core from the same patient showed strong positivity raises the question as too what factors determine which adenocarcinoma will show immunopositivity for AMACR.

\section{Duality of interest}

None declared.

\section{References}

1 Yang XJ, Laven B, Tretiakova M, et al. Detection of alpha-methylacyl-coenzyme A racemase in postradiation prostatic adenocarcinoma. Urology 2003;62: 282-286.

2 Beach R, Gown AM, de Peralta-Venturina MN, et al. P504S immunohistochemical detection in 405 prostatic specimens including 376 18-gauge needle biopsies. Am J Surg Pathol 2002;26:1588-1596.

3 Evans AJ. Methylacyl CoA racemase (p504s): overview and potential uses in diagnostic pathology as applied to prostate needle biopsies. Am J Clin Pathol 2003;56: 892-897.

4 Molinie V, Fromont G, Sibony M, et al. Diagnostic utility of a p63/ $\alpha$-methyl-CoA-racemase (p504s) cocktail in atypical foci in the prostate. Mod Pathol 2004;17: 1180-1190.

5 Yang XJ, Wu CL, Woda BA, et al. Expression of alpha methylacyl-CoA racemase (p504s) in atypical adenomatous hyperplasia of prostate. Am J Surg Pathol 2002;26: 921-925. 